Wisam Alame and W. M. ZająCZKowski (Warszawa)

\title{
GLOBAL EXISTENCE OF SOLUTIONS FOR INCOMPRESSIBLE MAGNETOHYDRODYNAMIC EQUATIONS
}

Abstract. Global-in-time existence of solutions for incompressible magnetohydrodynamic fluid equations in a bounded domain $\Omega \subset \mathbb{R}^{3}$ with the boundary slip conditions is proved. The proof is based on the potential method. The existence is proved in a class of functions such that the velocity and the magnetic field belong to $W_{p}^{2,1}(\Omega \times(0, T))$ and the pressure $q$ satisfies $\nabla q \in L_{p}(\Omega \times(0, T))$ for $p \geq 7 / 3$.

1. Introduction. In a bounded domain $\Omega \subset \mathbb{R}^{3}$ with boundary $S$ we consider the initial-boundary value problem for the equations of incompressible magnetohydrodynamics (see $[4,7]$ )

$$
\begin{array}{ll}
\partial_{t} v+v \cdot \nabla v+\nabla\left(q+H^{2} / 2\right) & \\
\quad-H \cdot \nabla H-\nu \Delta v=f & \text { in } \Omega^{T}=\Omega \times(0, T), \\
\operatorname{div} v=0 & \text { in } \Omega^{T}, \\
\partial_{t} H+v \cdot \nabla H-H \cdot \nabla v-\nu_{\sigma} \Delta H=0 & \text { in } \Omega^{T}, \\
\operatorname{div} H=0 & \text { in } \Omega^{T}, \\
\bar{n} \cdot D(v) \cdot \bar{\tau}_{\alpha}+\gamma v \cdot \bar{\tau}_{\alpha}=0 & \text { in } \Omega^{T}, \\
v \cdot \bar{n}=0 & \text { on } S^{T}=S \times(0, T), \\
H=0 & \text { on } S^{T}, \\
\left.v\right|_{t=0}=v(0) & \text { in } \Omega, \\
\left.H\right|_{t=0}=H(0) & \text { in } \Omega,
\end{array}
$$

2000 Mathematics Subject Classification: 35A05, 76W05, 76D03.

Key words and phrases: global existence, magnetohydrodynamic incompressible fluid, global-in-time existence.

Research of W. M. Zajączkowski supported by KBN Grant No. 2 P03A 00223. 
where $v=v(x, t)$ is the velocity of the fluid, $H=H(x, t)$ the magnetic field, $f=f(x, t)$ the external force, $\bar{n}$ the unit outward vector normal to $S, \bar{\tau}_{\alpha}$, $\alpha=1,2$, tangent vectors to $S, q=q(x, t)$ the pressure, $\gamma>0$ the constant slip coefficient. Moreover, $D(v)=\left\{v_{i, x_{j}}+v_{j, x_{i}}\right\}_{i, j=1,2,3}$ is the dilatation tensor.

The aim of this paper is to prove the global-in-time existence of solutions to (1.1) with small data in the $L_{p}$-approach.

Now we recall some results concerning mathematical questions of equations of magnetohydrodynamics (mhd). The first results on global existence of weak solutions to various initial-boundary value problems for mhd equations were given in $[5,6]$. In these papers global existence of strong solutions in $2 \mathrm{~d}$ and in the axially symmetric case was also proved. Moreover, global existence of regular solutions for small data was obtained.

In [8] existence, regularity and global properties of solutions of mhd equations such as global estimates, invariant sets, attracting sets have been obtained.

In $[9,10]$ by applying the semigroup technique global existence of regular solutions of mhd equations was proved under either smallness assumptions or some geometrical restrictions ( $2 \mathrm{~d}$, axially symmetric case).

Finally in [11] Stupialis has proved the existence of local solutions to the mhd equations such that the displacement term is taken into account.

In this paper we present a very simple and short proof of existence of global regular solutions to problem (1.1).

The main result can be stated as follows

Theorem. Let $f \in L_{p}\left(\Omega^{T}\right), f(0) \in L_{2}(\Omega), p \geq 7 / 3$, and let $(v(0), H(0))$ belong to $W_{p}^{2-2 / p}(\Omega)$. Assume that $\|f(t)\|_{L_{2}(\Omega)} \leq\|f(0)\|_{L_{2}(\Omega)} e^{-\lambda t}$ for some $\lambda>0$ and $f(t)$ describes dependence on time only. Let

$$
A=\|f\|_{L_{p}\left(\Omega^{T}\right)}+\|v(0)\|_{W_{p}^{2-2 / p}(\Omega)}+\|H(0)\|_{W_{p}^{2-2 / p}(\Omega)} .
$$

Assume that $A$ is so small that $c T^{1 / p} \leq 1$. Assume also that $S \in C^{2}$. Then there exists a solution for problem (1.1) such that $(v, H) \in W_{p}^{2,1}\left(\Omega^{T}\right)$, $\nabla q \in L_{p}\left(\Omega^{T}\right)$ and the following estimate holds:

$$
\begin{aligned}
& \|v\|_{W_{p}^{2,1}\left(\Omega_{k}\right)}+\|H\|_{W_{p}^{2,1}\left(\Omega_{k}\right)}+\|\nabla q\|_{L_{p}\left(\Omega_{k}\right)} \\
& \quad \leq c\left(\|f\|_{L_{p}\left(\Omega \times(k-1) T_{0},(k+1) T_{0}\right)}+\|v(k)\|_{W_{p}^{2-2 / p}(\Omega)}+\|H(k)\|_{W_{p}^{2-2 / p}(\Omega)}\right),
\end{aligned}
$$

where $\Omega_{k}=\Omega \times\left(k T_{0},(k+1) T_{0}\right)$ for $k \in \mathbb{N}, T_{0}>0$ and $c$ is independent of time.

2. Notation and auxiliary results. In our considerations we will need anisotropic Sobolev spaces $W_{p}^{m, n}\left(\Omega^{T}\right)$, where $m, n \in \mathbb{R}_{+} \cup\{0\}, p \geq 1$, and 
$\Omega^{T}=\Omega \times(0, T)$ with the norm

$$
\|v\|_{W_{p}^{m, n}\left(\Omega^{T}\right)}^{p}=\|v\|_{W_{p}^{m, 0}\left(\Omega^{T}\right)}^{p}+\|v\|_{W_{p}^{0, n}\left(\Omega^{T}\right)}^{p}
$$

where

$$
\|v\|_{W_{p}^{m, 0}\left(\Omega^{T}\right)}^{p}=\int_{0}^{T}\|v\|_{W_{p}^{m}(\Omega)}^{p} d t, \quad\|v\|_{W_{p}^{0, n}\left(\Omega^{T}\right)}^{p}=\int_{\Omega}\|v\|_{W_{p}^{n}(0, T)}^{p} d x
$$

for

$$
\begin{aligned}
\|v\|_{W_{p}^{m}(\Omega)}^{p} & =\sum_{|\alpha| \leq[m]}\left\|D_{x}^{\alpha} v\right\|_{L_{p}(\Omega)}^{p}+\sum_{|\alpha|=[m]} \iint_{\Omega} \frac{\left|D_{x}^{\alpha} v(x, t)-D_{y}^{\alpha} v(y, t)\right|^{p}}{|x-y|^{s+p(m-[m])}} d x d y, \\
\|v\|_{W_{p}^{n}(0, T)}^{p} & =\sum_{|\beta| \leq[n]}\left\|D_{t}^{\beta} v\right\|_{L_{p}(0, T)}^{p}+\sum_{|\beta|=[n]} \int_{0}^{T} \int_{0}^{T} \frac{\left|D_{t}^{\beta} v(x, t)-D_{t^{\prime}}^{\beta} v\left(x, t^{\prime}\right)\right|^{p}}{\left|t-t^{\prime}\right|^{1+p(n-[n])}} d t d t^{\prime},
\end{aligned}
$$

where $s \equiv \operatorname{dim} \Omega ;[m]$ is the integral part of $m ; D^{\alpha}$ is the derivative in the distributional sense; $D_{x}^{\alpha} \equiv \partial_{x_{1}}^{\alpha_{1}} \ldots \partial_{x_{s}}^{\alpha_{s}} ; \alpha=\left(\alpha_{1}, \ldots, \alpha_{s}\right)$ is a multiindex.

We will use the following results.

Lemma 2.1 ([2]). Let $f \in L_{p}\left(\Omega^{T}\right), G \in W_{p}^{1,0}\left(\Omega^{T}\right)$ and $p \geq 2$. Assume that there exist functions $A, B \in L_{p}\left(\Omega^{T}\right)$ such that $\partial_{t} G-\operatorname{div} f=\operatorname{div} B+A$ and diamsupp $A<2 \lambda_{1}$ for sufficiently small $\lambda_{1}>0$. Let $v(0) \in W_{p}^{2-2 / p}(\Omega)$, $b \in W_{p}^{1-1 / p, 1 / 2-1 / 2 p}\left(S^{T}\right), b_{3} \in W_{p}^{2-1 / p, 1-1 / 2 p}\left(S^{T}\right)$, where $b \equiv\left(b_{1}, b_{2}, 0\right)^{T}$. Then there exists a solution of the problem

$$
\begin{aligned}
& \partial_{t} v-\nu \Delta v+\nabla q=f \\
& \operatorname{div} v=G \\
& \bar{n} \cdot D(v) \cdot \bar{\tau}_{\alpha}+\left.\gamma v \cdot \bar{\tau}_{\alpha}\right|_{S^{T}}=b_{\alpha} \quad(\alpha=1,2), \\
& \left.v \cdot \bar{n}\right|_{S^{T}}=b_{3} \\
& \left.v\right|_{t=0}=v(0),
\end{aligned}
$$

such that $v \in W_{p}^{2,1}\left(\Omega^{T}\right), \nabla q \in L_{p}\left(\Omega^{T}\right)$, and the following estimate holds:

$$
\begin{aligned}
\|v\|_{W_{p}^{2,1}\left(\Omega^{T}\right)}+\|\nabla q\|_{L_{p}\left(\Omega^{T}\right)} \leq & c(T)\left(\|f\|_{L_{p}\left(\Omega^{T}\right)}+\|B\|_{L_{p}\left(\Omega^{T}\right)}+\lambda_{1}\|A\|_{L_{p}\left(\Omega^{T}\right)}\right. \\
& +\|G\|_{W_{p}^{1,0}\left(\Omega^{T}\right)}+\|b\|_{W_{p}^{1-1 / p, 1 / 2-1 / 2 p}\left(S^{T}\right)} \\
& \left.+\left\|b_{3}\right\|_{W_{p}^{2-1 / p, 1-1 / 2 p}\left(S^{T}\right)}+\|v(0)\|_{W_{p}^{2-2 / p}(\Omega)}\right)
\end{aligned}
$$

where $c(T)$ is an increasing positive function of $T$.

Lemma 2.2. Let $u, v \in W_{p}^{2,1}\left(\Omega^{T}\right)$ and $u(0), v(0) \in W_{p}^{2-2 / p}(\Omega), \Omega \subset \mathbb{R}^{3}$. Assume that $p \geq 7 / 3$. Then 


$$
\begin{aligned}
& \|u \cdot \nabla v\|_{L_{p}\left(\Omega^{T}\right)} \leq c T^{2 / p} \sup _{t}\|u\|_{W_{p}^{2-2 / p}(\Omega)} \sup \|v\|_{W_{p}^{2-2 / p}(\Omega)} \\
& \leq c T^{2 / p}\left(\|u\|_{W_{p}^{2,1}\left(\Omega^{T}\right)}+\|u(0)\|_{W_{p}^{2-2 / p}(\Omega)}\right)\left(\|v\|_{W_{p}^{2,1}\left(\Omega^{T}\right)}+\|v(0)\|_{W_{p}^{2-2 / p}(\Omega)}\right),
\end{aligned}
$$

where $c$ does not depend on $T$.

3. Existence. To prove the local existence we utilize the following method of successive approximations:

$$
\begin{aligned}
& \partial_{t} v_{n}-\nu \Delta v_{n}+\nabla q_{n} \\
& \quad=f-v_{n-1} \cdot \nabla v_{n-1}+H_{n-1} \cdot \nabla H_{n-1}-\nabla\left(H_{n-1}^{2} / 2\right), \\
& \operatorname{div} v_{n}=0, \\
& \partial_{t} H_{n}-\nu_{\sigma} \Delta H_{n}=H_{n-1} \cdot \nabla v_{n-1}-v_{n-1} \cdot \nabla H_{n-1}, \\
& \operatorname{div} H_{n}=0 \\
& \bar{n} \cdot D\left(v_{n}\right) \cdot \bar{\tau}_{\alpha}+\gamma v_{n} \cdot \bar{\tau}_{\alpha}=0, \quad \alpha=1,2, \\
& \left.\bar{n} \cdot v_{n}\right|_{S}=0, \\
& \left.H_{n}\right|_{S}=0, \\
& \left.v_{n}\right|_{t=0}=v(0),\left.\quad H_{n}\right|_{t=0}=H(0),
\end{aligned}
$$

and $v_{0}=H_{0}=0$.

LEMMA 3.1. Assume that $f \in L_{p}\left(\Omega^{T}\right), v(0) \in W_{p}^{2-2 / p}(\Omega), H(0) \in$ $W_{p}^{2-2 / p}(\Omega), p \geq 7 / 3$. Then there exists $T_{0}>0$ such that for all $T \leq T_{0}$ system (1.1) has a unique solution $v \in W_{p}^{2,1}\left(\Omega^{T}\right), H \in W_{p}^{2,1}\left(\Omega^{T}\right), \nabla q \in$ $L_{p}\left(\Omega^{T}\right)$, and the following estimate holds:

$$
\begin{aligned}
& \|v\|_{W_{p}^{2,1}\left(\Omega^{T}\right)}+\|H\|_{W_{p}^{2,1}\left(\Omega^{T}\right)}+\|\nabla q\|_{L_{p}\left(\Omega^{T}\right)} \\
& \quad \leq c(T)\left(\|f\|_{L_{p}\left(\Omega^{T}\right)}+\|v(0)\|_{W_{p}^{2-2 / p}(\Omega)}+\|H(0)\|_{W_{p}^{2-2 / p}(\Omega)}\right) .
\end{aligned}
$$

Proof. Let

$$
\begin{aligned}
X_{k}(T) & =\left\|v_{k}\right\|_{W_{p}^{2,1}\left(\Omega^{T}\right)}+\left\|H_{k}\right\|_{W_{p}^{2,1}\left(\Omega^{T}\right)}, \\
d(T) & =\|v(T)\|_{W_{p}^{2-2 / p}(\Omega)}+\|H(T)\|_{W_{p}^{2-2 / p}(\Omega)} .
\end{aligned}
$$

In view of Lemmas 2.1, 2.2 and the imbeddings $W_{p}^{2,1}\left(\Omega^{T}\right) \subset L_{q_{1}}\left(\Omega^{T}\right)$, $\nabla W_{p}^{2,1}\left(\Omega^{T}\right) \subset L_{q_{2}}\left(\Omega^{T}\right), W_{p}^{2-2 / p}(\Omega) \subset L_{q_{3}}(\Omega), \nabla W_{p}^{2-2 / p}(\Omega) \subset L_{q_{4}}(\Omega)$ with $5 / p-5 / q_{1} \leq 2,5 / p-5 / q_{2} \leq 1,5 / p-3 / q_{3} \leq 2,5 / p-3 / q_{4} \leq 1$ (see $[1,3])$ we have

$$
X_{n}(T) \leq c T^{1 / p}\left(X_{n-1}^{2}(T)+d^{2}(0)\right)+c\left(\|f\|_{L_{p}\left(\Omega^{T}\right)}+d(0)\right) .
$$

Suppose that

$$
X_{n-1}(T) \leq A
$$


and

$$
c T^{1 / p}\left(A^{2}+d^{2}(0)\right)+c\left(\|f\|_{L_{p}\left(\Omega^{T}\right)}+d(0)\right) \leq A
$$

Then we have the estimate

$$
X_{n}(T) \leq A
$$

for all $n \in \mathbb{N}$.

To satisfy condition (3.5) we assume

$$
c T^{1 / p} A \leq 1 / 2
$$

and

$$
c T^{1 / p} d^{2}(0)+c\left(\|f\|_{L_{p}\left(\Omega^{T}\right)}+d(0)\right) \leq \frac{1}{2} A .
$$

Then for small $A$ we have $T \leq(1 / 2 c A)^{p}$, and then by (3.8), the data must be suitably small.

To show convergence we introduce the differences $\widetilde{v}_{n}=v_{n}-v_{n-1}, \widetilde{H}_{n}=$ $H_{n}-H_{n-1}, \widetilde{q}_{n}=q_{n}-q_{n-1}$. They satisfy the following system of equations for $n \geq 2$ :

$$
\begin{aligned}
& \widetilde{v}_{n}-\nu \Delta \widetilde{v}_{n}+\nabla \widetilde{q}_{n}=-\left(\widetilde{v}_{n-1} \cdot \nabla v_{n-1}+v_{n-2} \cdot \nabla \widetilde{v}_{n-1}\right) \\
& -\left(\widetilde{H}_{n-1} \cdot \nabla H_{n-1}+H_{n-2} \cdot \nabla \widetilde{H}_{n-1}\right) \\
& -\left(\widetilde{H}_{n-1 i} \nabla H_{n-1 i}+H_{n-2 i} \nabla \widetilde{H}_{n-1 i}\right) \\
& \operatorname{div} \widetilde{v}_{n}=0 \text {, } \\
& \partial_{t} \widetilde{H}_{n}-\nu_{\sigma} \Delta \widetilde{H}_{n}=\widetilde{H}_{n-1} \cdot \nabla v_{n-1}+H_{n-2} \cdot \nabla \widetilde{v}_{n-1} \\
& -\left(\widetilde{v}_{n-2} \cdot \nabla H_{n-1}+v_{n-2} \cdot \nabla \widetilde{H}_{n-1}\right), \\
& \operatorname{div} \widetilde{H}_{n}=0 \text {, } \\
& \widetilde{v}_{n} \cdot \bar{n}=0 \text {, } \\
& \bar{n} \cdot D\left(\widetilde{v}_{n}\right) \cdot \bar{\tau}_{\alpha}+\gamma \widetilde{v}_{n} \cdot \bar{\tau}_{\alpha}=0, \\
& \widetilde{H}_{n}=0 \text {, } \\
& \left.\widetilde{v}_{n}\right|_{t=0}=0,\left.\quad \widetilde{H}_{n}\right|_{t=0}=0,
\end{aligned}
$$

where the summation over $i$ is assumed.

Let us introduce

$$
\Gamma_{n}(T)=\left\|\widetilde{v}_{n}\right\|_{W_{p}^{2,1}\left(\Omega^{T}\right)}+\left\|\widetilde{H}_{n}\right\|_{W_{p}^{2,1}\left(\Omega^{T}\right)} .
$$

From (3.9) we obtain

$$
\Gamma_{n}(T) \leq c T^{1 / p} A \Gamma_{n-1}(T)
$$

Hence for $c T^{2 / p} A<1$ we have convergence. This ends the proof. 
To prove the global existence we have to control the initial data in order to be able to apply Lemma 3.1.

Lemma 3.2. Assume that $f \in L_{p}\left(\Omega^{T}\right), f(0) \in L_{2}(\Omega), \Omega$ is a bounded domain, and let $\|f\|_{L_{2}(\Omega)} \leq\|f(0)\|_{L_{2}(\Omega)} e^{-\lambda t}, \lambda>0$. Assume that the Korn inequality (3.13) is valid. Then the following decay estimate holds:

$$
\|v\|_{L_{2}(\Omega)}^{2}+\|H\|_{L_{2}(\Omega)}^{2} \leq c e^{-c_{0} t} \quad \text { for } c_{0}>0 .
$$

Proof. Multiplying (1.1) 1 by $v$ and $(1.1)_{3}$ by $H$, adding, integrating over $\Omega$ and using the boundary conditions we obtain

$$
\begin{aligned}
\frac{d}{d t}\left(\|v\|_{L_{2}(\Omega)}^{2}+\| H\right. & \left.\|_{L_{2}(\Omega)}^{2}\right)+\nu_{\sigma}\|\nabla H\|_{L_{2}(\Omega)}^{2} \\
& +\nu\|D(v)\|_{L_{2}(\Omega)}^{2}+\gamma\|v \cdot \bar{\tau}\|_{L_{2}(S)}^{2}=\int_{\Omega} f \cdot v d x .
\end{aligned}
$$

Assume that we have the Korn inequality

$$
\|v\|_{H^{1}(\Omega)}^{2} \leq c\|D(v)\|_{L_{2}(\Omega)}^{2} .
$$

Then (3.12) implies

$$
\begin{aligned}
\frac{d}{d t}\left(\|v\|_{L_{2}(\Omega)}^{2}+\|H\|_{L_{2}(\Omega)}^{2}\right)+\nu^{\prime}\left(\|v\|_{L_{2}(\Omega)}^{2}\right. & \left.+\|H\|_{L_{2}(\Omega)}^{2}\right) \\
& \leq c\|f\|_{L_{2}(\Omega)}\|v\|_{L_{2}(\Omega)},
\end{aligned}
$$

where $\nu^{\prime}=\min \left\{\nu, \nu_{\sigma}\right\}$.

Let

$$
\alpha(t)=\|v(t)\|_{L_{2}(\Omega)}^{2}+\|H(t)\|_{L_{2}(\Omega)}^{2} .
$$

Then (3.14) implies

$$
\frac{d}{d t}\left(\alpha(t) e^{\nu^{\prime} t}\right) \leq c\|f(t)\|_{L_{2}(\Omega)}^{2} e^{\nu^{\prime} t} .
$$

Integrating (3.15) with respect to time gives

$$
\alpha(t) \leq c e^{-\nu^{\prime} t} \int_{0}^{t}\left\|f\left(t^{\prime}\right)\right\|_{L_{2}(\Omega)}^{2} e^{\nu^{\prime} t^{\prime}} d t^{\prime}+e^{-\nu^{\prime} t} \alpha(0) .
$$

Using the decay assumption

$$
\|f(t)\|_{L_{2}(\Omega)} \leq\|f(0)\|_{L_{2}(\Omega)} e^{-\lambda t}
$$

we obtain

$$
\alpha(t) \leq c e^{-2 \lambda t}\|f(0)\|_{L_{2}(\Omega)}^{2}+e^{-\nu^{\prime} t} \alpha(0) .
$$

This ends the proof.

REMARK 3.3. If (3.13) does not hold, we obtain from (3.12) the inequality

$$
\frac{d}{d t}\left(\|v\|_{L_{2}(\Omega)}^{2}+\|H\|_{L_{2}(\Omega)}^{2}\right)^{1 / 2} \leq c\|f\|_{L_{2}(\Omega)}
$$


SO

(3.18)

$$
\begin{aligned}
\|v(t)\|_{L_{2}(\Omega)}+\|H(t)\|_{L_{2}(\Omega)} \leq & c \int_{0}^{t}\left\|f\left(t^{\prime}\right)\right\|_{L_{2}(\Omega)} d t^{\prime} \\
& +\|v(0)\|_{L_{2}(\Omega)}+\|H(0)\|_{L_{2}(\Omega)} .
\end{aligned}
$$

Proof of the Theorem. To prove global existence we introduce a smooth function

$$
\zeta=\zeta\left(T_{1}, T_{2}, t\right)=\left\{\begin{array}{ll}
1 & \text { for } t \geq T_{1}, \\
0 & \text { for } t \leq T_{2},
\end{array} \quad T_{1}>T_{2} .\right.
$$

Let $\widetilde{v}=v \zeta, \widetilde{H}=H \zeta, \widetilde{q}=q \zeta, \widetilde{f}=f \zeta$. Then problem (1.1) takes the form

$$
\begin{aligned}
& \partial_{t} \widetilde{v}-\nu \Delta \widetilde{v}+\nabla \widetilde{q}=\widetilde{f}-v \cdot \nabla \widetilde{v}-H \cdot \nabla \widetilde{H}-H_{i} \nabla \widetilde{H}_{i}+v \dot{\zeta}, \\
& \operatorname{div} \widetilde{v}=0 \\
& \partial_{t} \widetilde{H}-\nu_{\sigma} \Delta \widetilde{H}=H \cdot \nabla \widetilde{v}-v \cdot \nabla \widetilde{H}+H \dot{\zeta} \\
& \operatorname{div} \widetilde{H}=0 \\
& \left.\widetilde{v} \cdot \bar{n}\right|_{s}=0 \\
& \bar{n} \cdot D(\widetilde{v}) \cdot \bar{\tau}_{\alpha}+\left.\gamma \widetilde{v} \cdot \bar{\tau}_{\alpha}\right|_{s}=0 \\
& \left.\widetilde{H}\right|_{s}=0, \\
& \left.\widetilde{v}\right|_{t=0}=0,\left.\quad \widetilde{H}\right|_{t=0}=0
\end{aligned}
$$

where $|\dot{\zeta}| \leq c /\left(T_{1}-T_{2}\right)$ and summation over repeated indices is assumed.

Assume that we have proved local existence up to time $T>T_{1}$. Then from (3.19) we have

$$
\begin{aligned}
d(T) \equiv & \|\widetilde{v}(T)\|_{W_{p}^{2-2 / p}(\Omega)}+\|\widetilde{H}(T)\|_{W_{p}^{2-2 / p}(\Omega)} \\
\leq & c\left(\|\widetilde{v}\|_{W_{p}^{2,1}\left(\Omega^{T}\right)}+\|\widetilde{H}\|_{W_{p}^{2,1}\left(\Omega^{T}\right)}\right) \leq c\left(\|f\|_{L_{p}\left(\Omega^{T}\right)}+T^{2 / p} A^{2}\right) \\
& +\frac{1}{\left(T_{1}-T_{2}\right)^{2}} \int_{T_{2}}^{T_{1}}\left(\left\|v\left(t^{\prime}\right)\right\|_{L_{2}(\Omega)}+\left\|H\left(t^{\prime}\right)\right\|_{L_{2}(\Omega)}\right) d t^{\prime} \\
\leq & c\left(\|f\|_{L_{p}\left(\Omega \times\left(T_{2}, T_{1}\right)\right)}+(T)^{2 / p} A^{2}\right) \\
& +\frac{1}{\left(T_{1}-T_{2}\right)} \sup _{t}\left(\|v(t)\|_{L_{2}(\Omega)}+\|H(t)\|_{L_{2}(\Omega)}\right)
\end{aligned}
$$

Assuming that $T$ is large, $T_{1}-T_{2}$ small compared to $T$ but still large, and using the decay estimate for $f$ we can assume

$$
\begin{aligned}
& \|f\|_{L_{p}\left(\Omega \times\left(T_{2}, T_{1}\right)\right)}+\left(T_{1}-T_{2}\right)^{2 / p} A^{2} \\
& \quad+\frac{1}{T_{1}-T_{2}} \sup _{t \in\left(T_{2}, T_{1}\right)}\left(\|v(t)\|_{L_{2}(\Omega)}+\|H(t)\|_{L_{2}(\Omega)}\right) \leq d(0) .
\end{aligned}
$$


This enables continuation of the local solution. For any $k \in \mathbb{N}$ and $T_{0}=$ $T_{1}-T_{2}$ we have (see Lemma 3.2 , Remark 3.3 )

$$
\begin{aligned}
& \|f\|_{L_{p}\left(\Omega_{k}\right)}+\left(T_{1}-T_{2}\right)^{2 / p} A^{2} \\
& \quad+\frac{1}{\left(T_{1}-T_{2}\right)} \sup _{t \in\left(k T_{0},(k+1) T_{0}\right)}\left(\|v(t)\|_{L_{2}(\Omega)}+\|H(t)\|_{L_{2}(\Omega)}\right) \leq d(0),
\end{aligned}
$$

where $\Omega_{k}=\Omega \times\left(k T_{0},(k+1) T_{0}\right)$. Hence

$$
\|v\|_{W_{p}^{2,1}\left(\Omega_{k}\right)}+\|H\|_{W_{p}^{2,1}\left(\Omega_{k}\right)} \leq c\left(\|f\|_{L_{p}\left(\Omega_{k}\right)}+d(0)\right)
$$

for sufficiently small initial data.

This ends the proof of existence of global solutions.

\section{References}

[1] R. A. Adams, Sobolev Spaces, Academic Press, New York, 1975.

[2] W. Alame, On existence of solutions for the nonstationary Stokes system with slip boundary conditions, Appl. Math., to appear.

[3] O. V. Besov, V. P. Il'in and S. M. Nikol'skiü, Integral Representation of Functions and Imbedding Theorems, Nauka, Moscow, 1975 (in Russian).

[4] T. G. Cowling, Magnetohydrodynamics, Interscience Tracts on Physics and Astronomy, New York, 1957.

[5] O. A. Ladyzhenskaya and V. A. Solonnikov, Solvability of some nonstationary problems of magnetohydrodynamics for viscous incompressible fluids, Trudy Mat. Inst. Steklov. 59 (1960), 115-173 (in Russian).

[6] - - - The linearization principle and invariant manifolds for magnetohydrodynamics equations, Zap. Nauchn. Sem. LOMI 38 (1973), 46-93 (in Russian).

[7] L. Landau and E. Lifschitz, Electrodynamics of Continuum Media, Mir, Moscow, 1986 (in Russian).

[8] M. Sermange and R. Temam, Some mathematical questions related to mhd-equations, Comm. Pure Appl. Math. 36 (1983), 635-664.

[9] G. Ströhmer, About the equations of non-stationary magneto-hydrodynamics with non-conducting boundaries, Nonlinear Anal. 39 (2000), 629- 647.

[10] G. Ströhmer and W. M. Zajączkowski, Existence and stability theorems for abstract parabolic equations, and some of their applications, in: Singularities and Differential Equations, Banach Center Publ. 33, Inst. Math., Polish Acad. Sci., Warszawa, 1996, 369-382.

[11] L. Stupialis, Initial-boundary value problems for the magnetohydrodynamics equations, Liet. Mat. Rink. 40 (2000), 228-254.

Institute of Mathematics

Warsaw University of Technology

Pl. Politechniki 1

00-661 Warszawa, Poland

E-mail: wisam@passnet.pl
Institute of Mathematics Polish Academy of Sciences Śniadeckich 8 00-956 Warszawa, Poland E-mail: wz@impan.gov.pl

Received on 29.9.2003;

revised version on 14.4.2004 